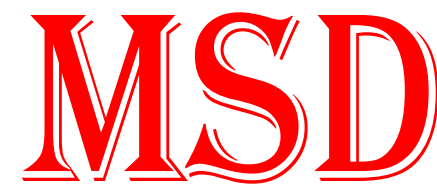

Medical Science and Discovery ISSN: 2148-6832

\section{Basal Cell Nevus Syndrome caused by a new splice site mutation in PTCH1}

\author{
Nattaya Nutsathapana ${ }^{1}$, Thareena Bunnag ${ }^{1}$, Prapaipit Chaowalit ${ }^{1}$, Chavalit \\ Supsrisunjai ${ }^{1}$ * \\ 1 Institute of Dermatology, Department of Medical Services, Ministry of Public Health, Bangkok, Thailand \\ * Corresponding Author: Chavalit Supsrisunjai E-mail: chervilius@hotmail.com
}

\section{ABSTRACT}

Objective: Basal cell nevus syndrome is a rare inherited autosomal dominant syndrome characterized by developmental defects and tumor predisposition. There are more than 400 reported PTCH1 mutations, including frameshift, nonsense, missense, deletions, duplications, and splicing mutations. We report a 68 -year-old Thai female presenting with multiple basal cell carcinoma scattered on the face and upper back and palmoplantar pits. Molecular diagnosis showed a novel heterozygous mutation in the splice site region c.746+1_746+4delGTAA, localized within the splice donor after exon 5 of PTCH1. Although the clinical manifestations are characteristic, this report adds another splice site mutation to the genotypic variation of BCNS patients and also highlights the importance of a multidisciplinary approach in the management of BCNS patients.

Keywords: Basal cell nevus syndrome, PTCH1, Mutation

\section{INTRODUCTION}

Basal cell nevus syndrome (BCNS) (OMIM109400) is a rare, multisystem, inherited autosomal dominant syndrome characterized by developmental defects and tumor predisposition (1). The clinical manifestations of BCNS include multiple basal cell carcinomas (BCCs), palmoplantar pits, odontogenic keratocysts of the jaw, ocular anomalies, skeletal, reproductive systems, and calcification of the falx cerebri (1). PTCH1 is the leading cause of mutations in BCNS (1). To date, there are more than 400 reported mutations, including frameshift, nonsense, missense, deletions, duplications, and splicing mutations (2). Genomic sequencing of 23 exons all showed mutation, yet there was no hot spot identified (2).

\section{CASE}

Here, we report a 68-year-old Thai female presenting with multiple progressive dark brownish to blackish lumps and bumps scattered on the face and upper back for about 30 years. Initially, the lesions developed on the periorbital and perioral areas. The patient reported no similar symptoms in family or relatives. Physical examination revealed multiple discrete well-defined dark brown to black papules and plaques with elevated rodent border and some ulcers scattered on both periorbital, perioral areas, temporal areas, forehead, and upper back (Fig. 1a, c). Bilateral symmetrical multiple pitted lesions were also noted her both palms and soles (Fig. 1b, d). Neither hepatosplenomegaly nor lymphadenopathy was observed. The histological exam revealed nodular and infiltrative basaloid tumor connecting from epidermis with peripheral nuclear palisading and peritumoral artificial clefts consistent with basal cell carcinoma (Fig. 1e). Additional laboratory tests for associated developmental anomalies and tumors were investigated, including chest radiograph and CT scan of the brain-skull-neck-oropharynx and whole abdomen. All the results were unremarkable. After obtaining informed consent, Sanger sequencing of all 23 coding exons and flanking introns of PTCH1 was performed using primers previously described (3) and genomic DNA from peripheral blood obtained from the affected individual, which revealed a novel heterozygous mutation, NM_001354919.1 c.746+1_746+4delGTAA, localized within the splice donor after exon (5). A prediction tool suggested alternate splicing leading to possible deleterious effects on the protein (https://franklin.genoox.com/clinical-db/home) (Fig. 1f). 


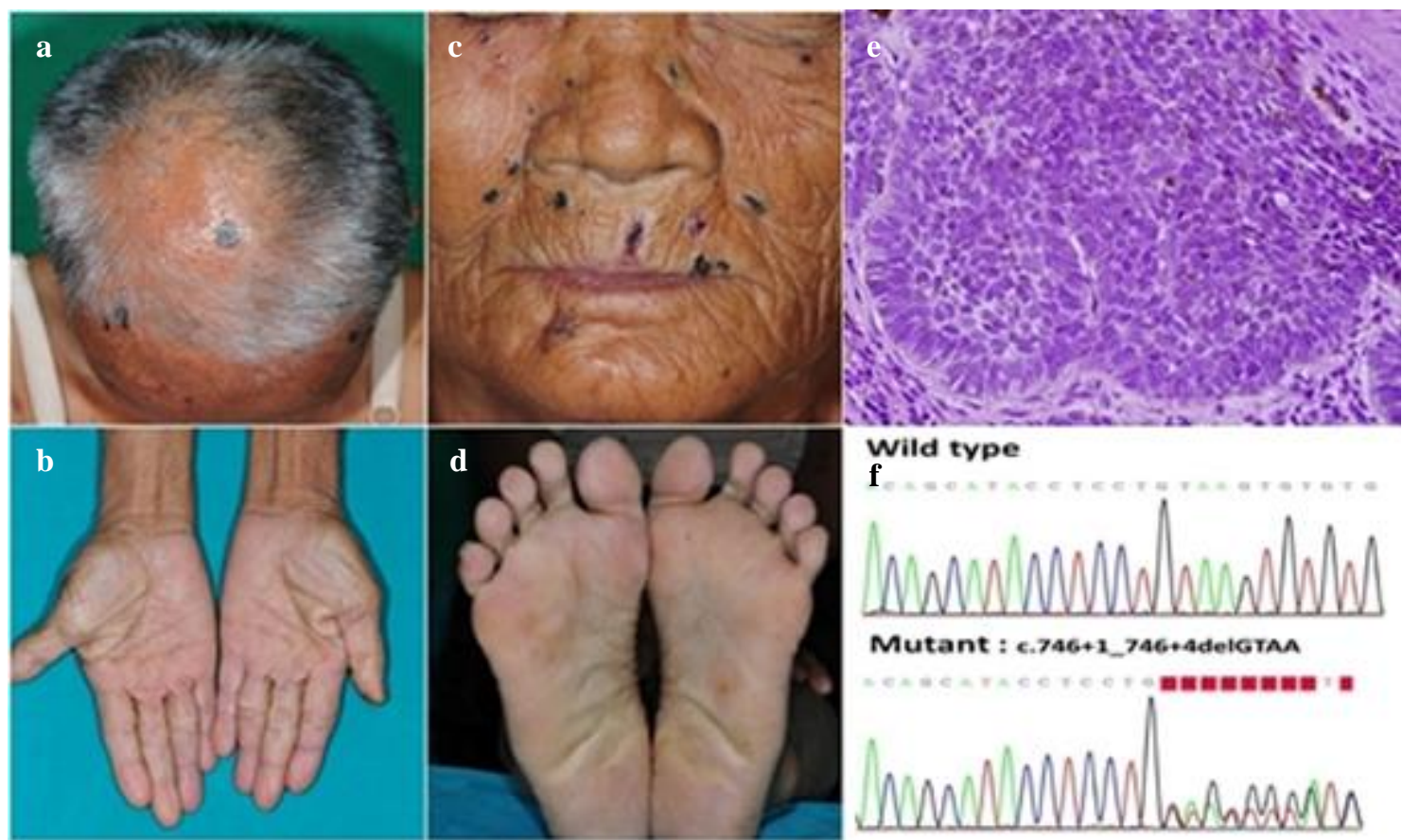

Figure 1. Clinicopathological and molecular findings in basal cell nevus syndrome. (a,c) multiple basal cell carcinoma on the head and neck; (b,d) numerous palmoplantar pits; (e) basal cell carcinoma showing basaloid tumor with peripheral nuclear palisading and peritumoral artificial clefts (hematoxylin\&eosin, 40x) (f) Sanger sequencing reveals a four base pairs heterozygous deletion in PTCH1 (NM_001354919.1; c.746+1_746+4delGTAA).

However, further functional studies will be required to confirm experimentally. This mutation does not appear in any lists of genomic databases, such as the gnomAD browser (https://gnomad.broadinstitute.org/). Thus, the molecular diagnosis was consistent with BCNS.

This syndrome exhibits a nearly complete penetrance with variable expressivity. The prevalence is estimated from $1 / 56,000$ up to $1 / 256,000$, in which both sexes are equally affected (4). Pathogenesis of BCNS has been attributed to heterozygous germline mutations in tumor suppressor gene PTCH1, located on chromosome 9q22.3 (4). The human PTCH1 gene encodes a transmembrane glycoprotein, patched 1 , which functions as the antagonist receptor for the sonic hedgehog ligand (4). Mutations in PTCH1 have been found in $40-80 \%$ of patients with BCNS (5). PTCH1 is the most frequently mutated gene, but mutations in PTCH2, SUFU can also occur5. About $20-30 \%$ of BCNS patients can be caused by de novo mutation (2).

The treatment of patients with BCNS requires a holistic approach. For the management of BCCs in BCNS, there are no established standard guidelines. Therefore, surgical excision by standard or micro excisional/Mohs micrographic surgery (MMS) has been the mainstay treatment4. BCCs lesions in our case were operated on by cryosurgery, and oral acitretin $25 \mathrm{mg} /$ day has been prescribed for cancer chemoprevention, as recommended for high-risk nonmelanoma skin cancer (4).

\section{CONCLUSION}

In summary, we report a case of BCNS in which clinical features fit into the diagnostic criteria with a novel heterozygous mutation in the splice site region. Although the clinical manifestation is characteristic, this report adds another splice site mutation, highlighting no evidence of genotype-phenotype correlation among BCNS patients.
Author contributions: NN, TB, PC, CS; Patient examination, Genetic analysis, Literature search and study design, data collection and analyzes CS; Writing article and revisions

Conflict of interest: The authors declared no potential conflicts of interest with respect to the research, authorship, and/or publication of this article. This research did not receive and specific grant from funding agencies in the public, commercial, or not-for-profit sectors.

Ethical issues: All authors declare originality of research.

\section{REFERENCES}

1. Kimonis VE, Goldstein AM, Pastakia B, Yang ML, Kase R, DiGiovanna JJ, Bale AE, Bale SJ. Clinical manifestations in 105 persons with nevoid basal cell carcinoma syndrome. American journal of medical genetics. 1997 Mar 31;69(3):299-308

2. Reinders MG, van Hout AF, Cosgun B, Paulussen AD, Leter EM, Steijlen PM, Mosterd K, van Geel M, Gille JJ. New mutations and an updated database for the patched-1 (PTCH 1) gene. Molecular genetics \& genomic medicine. 2018 May;6(3):409-15.

3. Sim YC, Kim GH, Choi SW, Ahn KM. Novel PTCH1 gene mutation in nevoid basal cell carcinoma syndrome. Journal of Craniofacial Surgery. 2018 May 1;29(3):e252-5

4. John AM, Schwartz RA. Basal cell naevus syndrome: an update on genetics and treatment. British journal of dermatology. 2016 Jan;174(1):68-76

5. Alonso N, Cañueto J, Ciria S, Bueno E, Palacios-Alvarez I, Alegre M, Badenas C, Barreiro A, Pena L, Maldonado C, Nespeira-Jato MV. Novel clinical and molecular findings in Spanish patients with naevoid basal cell carcinoma syndrome. British Journal of Dermatology. 2018 Jan;178(1):198-206.

Copyright (c) 2021 The Author(s); This is an open-access article distributed under the terms of the Creative Commons Attribution License (http://creativecommons.org/licenses/by/4.0), (CC BY NC) which permits unrestricted use, distribution, and reproduction in any medium, provided the original work is properly cited. International journal of Medical Science and Discovery. 